\title{
Book Review: \\ Research Methods in Human-Computer Interaction
}

\author{
Baris Mercimek \\ Anadolu University, Turkey
}

Research methods serve as a common tool for scholars in different disciplines. Successful and effective use of these methods within and across disciplines has been regarded as a crucial competence. The current resource, Research Methods in Human Computer Interaction, is among the pioneers and can be regarded as the leading source in terms of research in human computer interaction $(\mathrm{HCl})$. The book is written by Jonathan Lazar, Jinjuan Heidi and Harry Hochheiser (Wiley, 2010, 448 pages, ISBN13: 978-0470723371), and serves as a valuable starting point for $\mathrm{HCl}$ research methods.

The book is particularly prepared due to the perceived lack of a specific source for the scholars who conduct research on $\mathrm{HCl}$. More specifically, instead of addressing all popular qualitative and quantitative research and analysis methods, specific methods that are frequently used and adaptable to $\mathrm{HCl}$ have been considered.

The authors of the book are from a wide range of fields such as web usability, biomedical informatics and information security. To prevent possible difficulties in deciphering the contents and to facilitate reader-friendliness, some information about the format of the book was given at the inception. Except for some references to previous chapters, it is possible to go through the book through a non-sequential reading method. Besides, the research questions and exercises at the end of each chapter provides audience with guidance to build better associations among the topics.

Chapter 1 addresses the interdisciplinary nature of $\mathrm{HCl}$ as a field of study. In this regard, a broad view of the field is provided. The book is introduced as a practical reference book for appropriate and effective use of research methods in $\mathrm{HCl}$. The chapter compares and contrasts the $\mathrm{HCl}$ with various disciplines in terms of the methodological similarities, limitations, superiorities of the measurements, individual differences and validity concepts.

Chapter 2 focuses on the reasons of the emergence of experimental research studies. In this regard several concepts have been revisited such as hypothesis, dependent and independent variables, significance, randomization, type I and type II errors in the decision making processes. The chapter also provides the audience with some hints to prepare an experimental environment properly so that more accurate measurements are built and administered to sustain a desired degree of validity. Other matters discussed in the chapter can be listed as the importance of varying mindsets of the humans -who are basic subjects of the $\mathrm{HCl}-$, the format of assigning groups, and task assignments.

Chapter $\mathbf{3}$ is aimed to provide insight regarding an appropriate and valid hypothesis building in an experimental design along with test conditions and group arrangements. In addition, the 
effects of the interaction experience on the measurements are discussed. It is further emphasized that bias and error cannot be eliminated completely in an experimental endeavor, which requires $\mathrm{HCl}$ scholars to be careful while interpreting their findings. Finally, the representative capacity of the samples in both pilot and actual implementation is given utmost importance so that the findings can be generalizable to real settings.

Chapter 4 discusses the basic preparation for statistical analysis processes. Usage of parametric and non-parametric tests are presented in the chapter as well. The notions of relationship and effect were addressed with regard to different analysis procedures including t-tests, ANOVAs, correlation, regression and nonparametric methods. A particular importance is given to the interpretation process which begins with the assessment of the normal distribution assumption and ends with the discussion of core findings. It is further expressed that larger samples provide the researcher with results that are closer to the real situation in the universe.

Chapter 5 focuses on the surveys and their conceptualization. The chapter begins with the importance of data collection tools which allow researchers to obtain larger amounts of data. The authors list the prerequisites as building well-defined research questions and selecting suitable research participants. Unlike other fields in humanities, non-probabilistic sampling methods such as purposeful sampling are preferred in $\mathrm{HCl}$ studies. Authors further provide suggestions to maximize data, overcome problems in user interface design, and manage the data collection, preparation and analysis processes.

The book offers a comprehensive source for the use of quantitative and qualitative research methods in $\mathrm{HCl}$. Up to this point quantitative methods were discussed. Hints for qualitative research methods can be found in chapter 6 through chapter 9 in a reader-friendly flow. Definition of qualitative research methods and practical implications can be useful particularly for novice researchers.

Chapter 6 aims to address the use of researcher diaries. They may contain either superficial responses or in-depth response analyses. Diaries can be kept through word processing programs or web sites in the $\mathrm{HCl}$ field. They can also be used as a supportive tool in experimental studies to back up or substitute observations. The focal point of feedback diaries and the elicitation diaries are discussed in the chapter as well. Encouraging participants throughout the experimental process is considered important so that the continuity of the data collection is sustained and the experimental context is described better by the researcher. At the end of the chapter, the use of content analysis is recommended so that reasonable results can be obtained.

Chapter 7 addresses case studies related to authentic life experiences or unique events. The importance of data triangulation and selecting appropriate analysis methods is emphasized to conduct an extensive research. As in all qualitative endeavors, case studies cannot be generalized, but contain tips and implications for similar applications. Particularly in $\mathrm{HCl}$ studies, tips for the successful use of technology and discovering effective operations to apply to different areas can be considered as relevant instances. The most important elements of the research are regarded as unique and incomparable cases. Revealing meaningful patterns of the collected data, presentation of proof and gradual process are listed as further tasks for researchers. 
Interviews are considered as an incredible opportunity for $\mathrm{HCl}$ research. They can be used to reveal necessary corrections or problematic processes in a project or prototype. In this regard, Chapter 8 covers important topics pertaining to interviews such as group selection according to the research question, question structures, physical relevance, and the concordance among the analyses of different researchers. The authors further discuss the insufficiency of single type data to answer a research question. Then, supportive features of the interviews are discussed. As interviews constitute a psychological process, the roles of researchers to increase communication and the gradual structuring of the questions are further issues considered in the chapter.

Chapter 9 describes ethnographic studies which are often associated with social sciences. In this regard, the use of technology -particularly computers-, human, systems and process dimensions are mentioned. Using system and results of the interaction reflect the basic research situation in $\mathrm{HCl}$. Investigation of the interaction provided by the use of appliances is also discussed within the section.

Chapter 10 begins with the usability testing issue. In this process, users evaluate the system and their critical or recurrent comments are considered to improve the current system. Userbased, automated and expert-based tests are described as well. With Wizard-of-Oz test simulation, interface evaluation of the interaction of the human-machine and human-human dimensions are presented.

Objectivity, validity and reliability of qualitative data analysis issues are considered in Chapter 11. Grounded theory and experimental research relationships are presented. In terms of trustworthiness, appropriate hints for the triangulation of data, participants and researcher perspectives are provided. Analysis processes are summarized while presenting relevant and related parts as a meaningful whole. The chapter ends with addressing the role of automatic annotation applications while analyzing multimedia content.

Chapter 12 discusses the automated data collection tools preferred by many researchers. In this regard, user interaction throughout the system activity and the effectiveness in terms of research are evaluated. The authors further describes the hybrid approaches and indirect measurement issues. Even though the researcher and the participant are physically isolated from each other, the importance of sustaining the continuity of the researcher-participation confidence is addressed.

Chapter 13 focuses on evaluating the meaningful responses of the human body through physiological means. In this regard, use of eye tracking, galvanic skin response, blood pressurevolume, and electroencephalography is addressed within the context of the $\mathrm{HCl}$ research. These tools carry utmost importance as they support the psychological data and serve as a concrete evidence of the ongoing interaction processes. Due to complex psychological processes, interpreting physiological data is a critical issue so the interpretation of an observed data range in a specific time and through a specific sense should be properly operationalized.

Chapter 14 addresses the hints for working with human subjects and researchers' responsibilities towards participants. In terms of quantity and quality, suitability of the participants for the current research question is also emphasized. Sustaining a natural routine in the research process is underlined, and the importance of protecting participants' physical and psychological comfort is expressed. 
Chapter 15 can be considered effective and engaging while guiding the audience to work with research participants with impairments. Drawbacks of multiple participants are discussed as well. The authors further point out the arrangements to ensure maximum amount and variety of data. Working with special participants in $\mathrm{HCl}$ settings can also serve as a satisfactory endeavor and trigger further research.

Action research is a developing and effective method, which can be implemented in $\mathrm{HCl}$ endeavors as well. However, such a topic is not reflected in the current resource. Authors may focus on this methodology in subsequent editions so that a new research perspective is integrated into the current research agenda.

The book highlights the importance of using quantitative and qualitative research methods together to address research questions more effectively. It provides the audience with guidance in the selection of appropriate methods for issues related to $\mathrm{HCl}$. Even though provided examples are particularly associated with the $\mathrm{HCl}$, scholars in other disciplines are likely to be familiar with those examples as well. The book is devoted to a particular field of study; however, it resembles a social science research reference in many ways. Thus, it may serve both novice and expert researchers in related fields. In brief, the source is quite easy to read and useful for the application of research methods in $\mathrm{HCl}$. Actual implication samples and practical hints are particularly useful in transforming our theoretical background into practical solutions in the field.

Correspondence: Baris Mercimek, Doctoral Student, Department of Computer Education and Instructional Technologies, Graduate School of Educational Sciences, Anadolu University, Yunus Emre Campus, Eskisehir, Turkey 